\title{
On Burr III Marshal Olkin family: development, properties, characterizations and applications
}

Fiaz Ahmad Bhatti ${ }^{*}$, G. G. Hamedani ${ }^{2}$, Mustafa C. Korkmaz ${ }^{3}$, Gauss M. Cordeiro ${ }^{4}$, Haitham M. Yousof ${ }^{5}$ and Munir Ahmad ${ }^{1}$

\author{
*Correspondence: fiazahmad72@ \\ gmail.com \\ ${ }^{1}$ National College of Business \\ Administration and Economics, \\ Lahore, Pakistan \\ Full list of author information is \\ available at the end of the article
}

\begin{abstract}
In this paper, a flexible family of distributions with unimodel, bimodal, increasing, increasing and decreasing, inverted bathtub and modified bathtub hazard rate called Burr III-Marshal Olkin-G (BIIIMO-G) family is developed on the basis of the T-X family technique. The density function of the BIIIMO-G family is arc, exponential, left- skewed, right-skewed and symmetrical shaped. Descriptive measures such as quantiles, moments, incomplete moments, inequality measures and reliability measures are theoretically established. The BIIIMO-G family is characterized via different techniques. Parameters of the BIIIMO-G family are estimated using maximum likelihood method. A simulation study is performed to illustrate the performance of the maximum likelihood estimates (MLEs). The potentiality of BIIIMO-G family is demonstrated by its application to real data sets.
\end{abstract}

Keywords: Moments, Reliability, Maximum likelihood Estimation, Characterizations

\section{Introduction}

Marshall and Olkin (1997) developed a new family of distributions with an additional shape parameter called Marshall and Olkin-G (MO-G) family. The survival function of MO-G family is

$$
S(x)=\frac{\lambda \bar{G}(x, \psi)}{1-\bar{\lambda} \bar{G}(x, \psi)}, \lambda>0, \bar{\lambda}=1-\lambda, x \in \Re
$$

where $G(x, \psi)$ is the baseline cumulative distribution function(cdf) which may depend on the vector parameter $\psi$.

Many famous MO-G families and its special distributions are available in literature such as Marshall-Olkin-G (Marshall and Olkin; 1997), the MO extended Lomax (Ghitany et al.; 2007), MO semi-Burr and MO Burr (Jayakumar and Mathew; 2008), MO q-Weibull (Jose et al.; 2010), MO extended Lindley (Ghitany et al.; 2012), the generalized MO-G (Nadarajah et al. (2013), the MO Fréchet (Krishna et al; 2013), the MO family (Cordeiro and Lemonte; 2013), MO extended Weibull(Santos-Neto et al.; 2014), the beta MO-G (Alizadeh et al. 2015), the MO generalized exponential (Ristić, \& Kundu; 2015), MO gamma-Weibull (Saboor and Pogány; 2016), MO generalized-G

(c) The Author(s). 2019 Open Access This article is distributed under the terms of the Creative Commons Attribution 4.0 International License (http://creativecommons.org/licenses/by/4.0/), which permits unrestricted use, distribution, and reproduction in any medium, provided you give appropriate credit to the original author(s) and the source, provide a link to the Creative Commons license, and indicate if changes were made. 
(Yousof et al.; 2018), MO additive Weibull (Afify et al.; 2018) and Weibull MO family (Korkmaz et al.; 2019).

This paper is sketched into the following sections. In Section 2, BIIIMO-G family is development via the T-X family technique. The basic structural properties and sub-models are also studied. In Section 3, two special models are studied. Section 4, deals with linear representations for the cdf and pdf of the BIIIMO-G family. In Section 5, moments, incomplete moments, inequality measures and some other properties are theoretically derived. In Section 6, stress-strength reliability and multicomponent stress-strength reliability of the model are studied. In Section 7, BIIIMO-G family is characterized via (i) conditional expectation; (ii) ratio of truncated moments and (iii) reverse hazard rate function. In Section 8, the maximum likelihood method is employed to estimate the parameters of the Burr III Marshall Olkin Weibull (BIIIMO-W) and Burr III Marshall Olkin Lindley (BIIIMO-L) distributions. In section 9, a simulation study is performed to illustrate the performance of the maximum likelihood estimates (MLEs). In Section 10, the potentiality of BIIIMO-G family is demonstrated by its application to real data sets: survival times of leukemia patients and bladder cancer patients' data. Goodness of fit of the probability distribution through different methods is studied. Section 11 contains concluding remarks.

\section{Development of BIIIMO-G family}

Alzaatreh et al. (2013) proposed a T-X family technique for the development of the wider families based on any probability density function (pdf). The cdf of the T- $X$ family of distributions is given by

$$
F(x)=\int_{a_{1}}^{W[G(x ; \psi)]} r(t) d t, \quad x \in \mathbb{R}
$$

where $r(t)$ is the pdf of a random variable (rv) $T$, where $T \in\left[a_{1}, a_{2}\right]$ for $-\infty \leq a_{1}<a_{2}<\infty$ and $W[G(x ; \psi)]$ is a function of the baseline cumulative distribution function (cdf) of a rv $X$, depending on the vector parameter $\psi$ and satisfies three conditions i) $W[G(x ; \psi)] \in\left[a_{1}\right.$, $\left.a_{2}\right]$, ii) $W[G(x ; \psi)]$ is differentiable and monotonically increasing and iii) $\lim _{x \rightarrow-\infty} W[G(x ; \psi)]$ $\rightarrow a_{1}$ and $\lim _{x \rightarrow \infty}[G(x ; \psi)] \rightarrow a_{2}$. The pdf corresponding to (2) is

$$
f(x)=\left\{\frac{\partial}{\partial x} W[G(x ; \psi)]\right\} r\{W[G(x ; \psi)]\}, \quad x \in \mathbb{R} .
$$

In this article, the BIIMO-G family is developed via the T-X family technique by setting.

$$
r(t)=\alpha \beta t^{-\beta-1}\left\{1+t^{-\beta}\right\}^{-\alpha-1}, t>0, \alpha>0, \beta>0, \text { and } W[G(x ; \psi)]=-\log \left[\frac{\lambda \bar{G}(x, \psi)}{1-\bar{\lambda} \bar{G}(x, \psi)}\right] .
$$

Then, the cdf of BIIIMO-G family is

$$
F(x ; \alpha, \beta, \lambda, \psi)=\left\{1+\left[-\log \left(\frac{\lambda \bar{G}(x ; \psi)}{1-\bar{\lambda} \bar{G}(x ; \psi)}\right)\right]^{-\beta}\right\}^{-\alpha}, x \in \mathbb{R}
$$

where $\alpha>0, \beta>0, \lambda>0$ and $\psi>0$ are parameters.

The pdf corresponding to (4) is given by 


$$
\begin{array}{r}
f(x ; \alpha, \beta, \lambda, \psi)=\frac{\alpha \beta g(x ; \psi)}{\bar{G}(x ; \psi)(1-\bar{\lambda} \bar{G}(x ; \psi))}\left[-\log \left(\frac{\lambda \bar{G}(x ; \psi)}{1-\bar{\lambda} \bar{G}(x ; \psi)}\right)\right]^{-\beta-1} \times \\
\left\{1+\left[-\log \left(\frac{\lambda \bar{G}(x ; \psi)}{1-\bar{\lambda} \bar{G}(x ; \psi)}\right)\right]^{-\beta}\right\}^{-\alpha-1}, x \in \mathbb{R},
\end{array}
$$

where $g(x ; \psi)$ is the baseline pdf. In future, a rv with pdf (5) is denoted by $X \sim B I I I M O$ $G(\alpha, \beta, \lambda, \psi)$. The dependence on the parameter vector $\psi$ can be omitted and simply write as $g(x)=g(x ; \psi)$,

$G(x)=G(x ; \psi)$ and $f(x)=f(x ; \alpha, \beta, \lambda, \psi)$.

Let $T$ be a BIII random variable with shape parameters $\alpha, \beta$. The BIIIMO-G rv with cdf (4) can be obtained from

$$
\operatorname{Pr}(\mathrm{X}<x)=\operatorname{Pr}\left(T \leq-\log \left[\frac{\lambda \bar{G}(x)}{1-\bar{\lambda} \bar{G}(x)}\right]\right) .
$$

Hence, the rv $X=G^{-1}\left[\frac{\lambda-\lambda \exp (-T)}{\lambda+\bar{\lambda} \exp (-T)}\right]$ has the BIIIMO-G distribution. The quantile function (qf) of $\mathrm{X}$ is the solution of the non-linear equation

$$
x=Q(u)=Q_{G}\left(1-\left\{\bar{\lambda}+\lambda \exp \left[\left(u^{-\frac{1}{\alpha}}-1\right)^{-\frac{1}{\beta}}\right]\right\}^{-1}\right),
$$

where $Q_{G}()=.G^{-1}($.$) is the qf of the baseline distribution. Hence, if \mathrm{U}$ is a uniform rv on $(0,1)$, then $X=Q(U)$ follows the BIIIMO-G family.

\section{Transformations and compounding}

The BIIIMO-G family is derived through (i) ratio of the exponential and gamma random variables and (ii) compounding generalized inverse Weibull-MO (GIW-MO) and gamma distributions.

\section{Lemma}

i. Let the random variable $Z_{1}$ have the exponential distribution with parameter value 1 and the random variable $Z_{2}$ have the fractional gamma i.e., $Z_{2} \sim \operatorname{Gamma}(\alpha, 1)$. Then, for

$$
Z_{1}=\left[-\log \left(\frac{\lambda \bar{G}(x)}{1-\bar{\lambda} \bar{G}(x)}\right)\right]^{-\beta} Z_{2}
$$

we have

$$
X=G^{-1}\left\{\frac{\lambda+\lambda \exp \left[\left(\frac{Z_{2}}{Z_{1}}\right)^{\frac{1}{\beta}}\right]}{\bar{\lambda}+\lambda \exp \left[\left(\frac{Z_{2}}{Z_{1}}\right)^{\frac{1}{\beta}}\right]}\right\} \sim \operatorname{BIIIMO}-G(\alpha, \beta, \lambda, \psi)
$$


ii. If $Y \mid \beta, \lambda, \theta \sim \operatorname{GIWMO}(y ; \beta, \lambda, \theta)$ and $\theta \mid \alpha \sim \operatorname{gamma}(\theta ; \alpha)$, then integrating the effect of $\theta$ with the help of

$$
f(y, \alpha, \beta, \lambda)=\int_{0}^{\infty} g(y \mid \beta, \lambda, \theta) g(\theta \mid \alpha) d \theta,
$$

we have $Y \sim B I I I M O-G(\alpha, \beta, \lambda, \psi)$..

\section{Structural properties of BIIIMO-G family}

The survival, hazard, cumulative hazard, reverse hazard functions and the Mills ratio of a random variable $\mathrm{X}$ with BIIIMO-G family are, respectively given, by

$$
\begin{aligned}
& S(x)=1-\left\{1+\left[-\log \left(\frac{\lambda \bar{G}(x)}{1-\bar{\lambda} \bar{G}(x)}\right)\right]^{-\beta}\right\}^{-\alpha} \\
& h(x)=\frac{\frac{\alpha \beta g(x)}{\bar{G}(x)(1-\bar{\lambda} \bar{G}(x))}\left[-\log \left(\frac{\lambda \bar{G}(x)}{1-\bar{\lambda} \bar{G}(x)}\right)\right]^{-\beta-1}}{\left\{1+\left[-\log \left(\frac{\lambda \bar{G}(x)}{1-\bar{\lambda} \bar{G}(x)}\right)\right]^{-\beta}\right\}^{\alpha+1}-\left\{1+\left[-\log \left(\frac{\lambda \bar{G}(x)}{1-\bar{\lambda} \bar{G}(x)}\right)\right]^{-\beta}\right\}}, \\
& r(x)=\frac{d}{d x} \ln \left\{1+\left[-\log \left(\frac{\lambda \bar{G}(x)}{1-\bar{\lambda} \bar{G}(x)}\right)\right]^{-\beta}\right\}^{-\alpha}, \\
& H(x)=-\ln \left[1-\left\{1+\left[-\log \left(\frac{\lambda \bar{G}(x)}{1-\bar{\lambda} \bar{G}(x)}\right)\right]^{-\beta}\right\}^{-\alpha}\right]
\end{aligned}
$$

and

$$
m(x)=\frac{\left\{1+\left[-\log \left(\frac{\lambda \bar{G}(x)}{1-\bar{\lambda} \bar{G}(x)}\right)\right]^{-\beta}\right\}^{\alpha+1}-\left\{1+\left[-\log \left(\frac{\lambda \bar{G}(x)}{1-\bar{\lambda}(x)}\right)\right]^{-\beta}\right\}}{\alpha \beta \frac{\lambda g(x)}{\bar{G}(x)(1-\bar{\lambda} \bar{G}(x))}\left[-\log \left(\frac{\lambda \bar{G}(x)}{1-\bar{\lambda} \bar{G}(x)}\right)\right]^{-\beta-1}} .
$$

The elasticity $e(x)=\frac{d \ln \mathrm{F}(x)}{d \ln x}=x r(x)$ for BIIIMO-G family is

$$
e(x)=\frac{d}{d \ln x} \ln \left\{1+\left[-\log \left(\frac{\lambda \bar{G}(x)}{1-\bar{\lambda} \bar{G}(x)}\right)\right]^{-\beta}\right\}^{-\alpha} .
$$

The elasticity of BIIIMO-G family shows the behavior of the accumulation of probability in the domain of the random variable.

\section{Sub-models}

The BIIIMO-G family has the following sub models (Table 1). 
Table 1 Sub-models of BIIIMO distribution

\begin{tabular}{lllll}
\hline Variable & Parameters & & & Name of Distribution \\
\hline $\mathrm{X}$ & $a$ & $\beta$ & $\lambda$ & BIIIMO-G family \\
$\mathrm{X}$ & $a$ & 1 & $\lambda$ & Inverse Lomax MO-G family \\
$\mathrm{X}$ & 1 & $\beta$ & $\lambda$ & Log-logistic MO-G family \\
$\mathrm{X}$ & $a$ & $\beta$ & 1 & BIII-X family \\
$\mathrm{X}$ & $a$ & 1 & 1 & Inverse Lomax-X family \\
$\mathrm{X}$ & 1 & $\beta$ & 1 & Log-logistic-X family \\
$\mathrm{X}$ & 1 & $\beta$ & $\lambda$ & MO-G family \\
$\frac{1}{\mathrm{X}}$ & $a$ & $\beta$ & $\lambda$ & BXIIMO-G family \\
$\mathrm{X}$ & 1 & 1 & 1 & Base line distribution \\
\hline
\end{tabular}

\section{Special BIIIMO-G models}

The BIIIMO-G family density (5) produces greater flexibility than any baseline distribution for data modeling. It can be most tractable, when the functions $g(x ; \psi)$ and $G(x ; \psi)$ have simple analytic expressions. Then, two special sub-models of BIIIMO-G family are introduced.

\section{The BIIIMO-Weibull (BIIIMO-W) distribution}

The cdf and pdf of the Weibull random variable are $g(x ; b)=b x^{b-1} e^{-x^{b}}, x>0, b>0$ and $G(x ; b)=1-e^{-x^{b}}, x \geq 0, b>0$ where $\psi=b$. Then, the pdf of the BIIIMO-W model is given by

$$
\begin{aligned}
f(x ; \alpha, \beta, \lambda, b)=\frac{b \alpha \beta x^{b-1}}{\left(1-\bar{\lambda} e^{-x^{b}}\right)}[ & \left.\log \left(\frac{\lambda e^{-x^{b}}}{1-\bar{\lambda} e^{-x^{b}}}\right)\right]^{-\beta-1} \times \\
& \left\{1+\gamma\left[-\log \left(\frac{\lambda e^{-x^{b}}}{1-\bar{\lambda} e^{-x^{b}}}\right)\right]^{-\beta}\right\}^{-\alpha-1}, x>0,
\end{aligned}
$$

The following graphs show that shapes of BIIIMO-W density are arc, bimodal, exponential, left- skewed, right-skewed and symmetrical (Fig. 1). The BIIIMO-W distribution has uni-model, bimodal, increasing, increasing and decreasing, inverted bathtub and modified bathtub hazard rate function (hrf) Fig. 2.

\section{The Burr III Marshal Olkin-Lindley (BIIIMO-L) distribution}

The cdf and pdf of the Lindley random variable are $g(x ; \psi)=\frac{b^{2}}{(1+b)}(1+x) e^{-b x}, x>0, b>0$ and $G(x ; \psi)=1-\left(1+\frac{b x}{1+b}\right) e^{-b x}, x \geq 0$ where $\psi=b$. Then, the pdf of the BIIIMO-L model are given by

$$
\begin{array}{r}
f(x ; \alpha, \beta, \lambda, b)=\frac{\alpha \beta \frac{b^{2}}{(1+b)}(1+x) e^{-b x}}{\left(1+\frac{b x}{1+b}\right) e^{-b x}}\left\{-\log \left[\frac{\lambda\left(1+\frac{b x}{1+b}\right) e^{-b x}}{1-\bar{\lambda}\left(1+\frac{b x}{1+b}\right) e^{-b x}}\right]\right\}^{-\beta-1} \times \\
\left(1+\left\{-\log \left[\frac{\lambda\left(1+\frac{b x}{1+b}\right) e^{-b x}}{1-\bar{\lambda}\left(1+\frac{b x}{1+b}\right) e^{-b x}}\right]\right\}^{-\beta}, x>0,\right.
\end{array}
$$




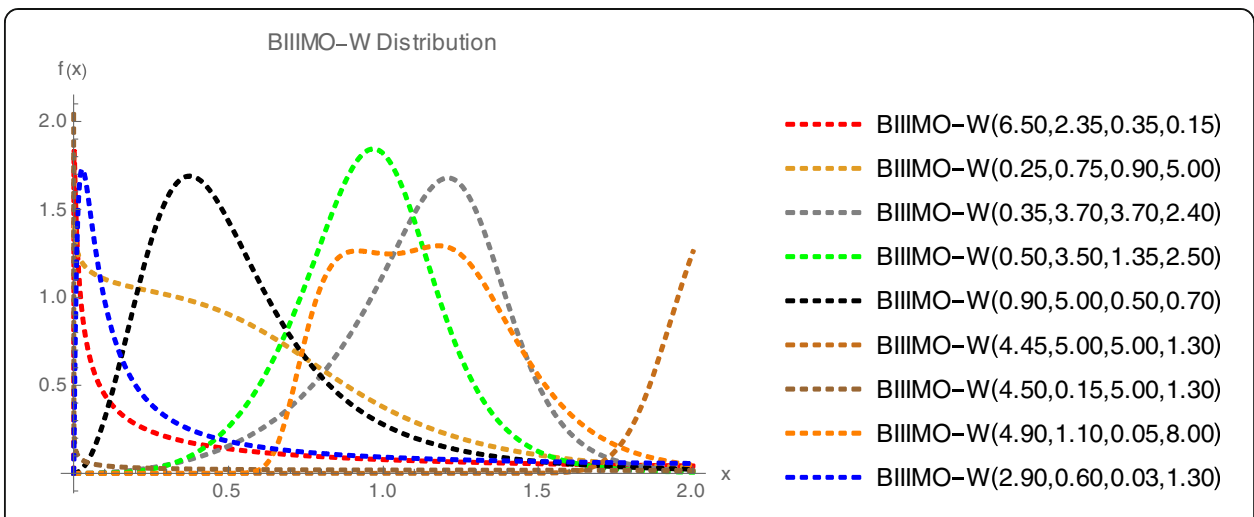

Fig. 1 Plot of pdf of BlIIMO-W distribution for selected parameter values

The following graphs show that shapes of BIIIMO-L density are J, revers J, arc, exponential, left- skewed, right-skewed and symmetrical (Fig. 3). The BIIIMO-L distribution has unimodal, increasing, increasing and decreasing, decreasing-increasing-decreasing inverted bathtub, bathtub and modified bathtub hazard rate function (Fig. 4).

\section{Useful expansions}

In this sub-section, the linear representations for the cdf and pdf of the BIIIMO-G family are obtained. The cdf in (4) can be expressed as

$$
F(x)=1-\sum_{i \geq 1}^{\infty}(-1)^{i+1}\left(\begin{array}{l}
\alpha+i-1 \\
i
\end{array}\left[-\log \left(\frac{\lambda \bar{G}(x)}{1-\bar{\lambda} \bar{G}(x)}\right)\right]^{-i \beta},\right.
$$

Following (Tahir et al. 2016), we have

$$
\left[-\log \left(\frac{\lambda \bar{G}(x)}{1-\bar{\lambda} \bar{G}(x)}\right)\right]^{-i \beta}=\frac{\sum_{k=0}^{\infty} p_{k}\left[1-\left(\frac{\lambda \bar{G}(x)}{1-\bar{\lambda} \bar{G}(x)}\right)\right]^{k}}{\sum_{k=0}^{\infty} q_{k}\left(1-\frac{\lambda \bar{G}(x)}{1-\bar{\lambda} \bar{G}(x)}\right)^{k}}
$$

where

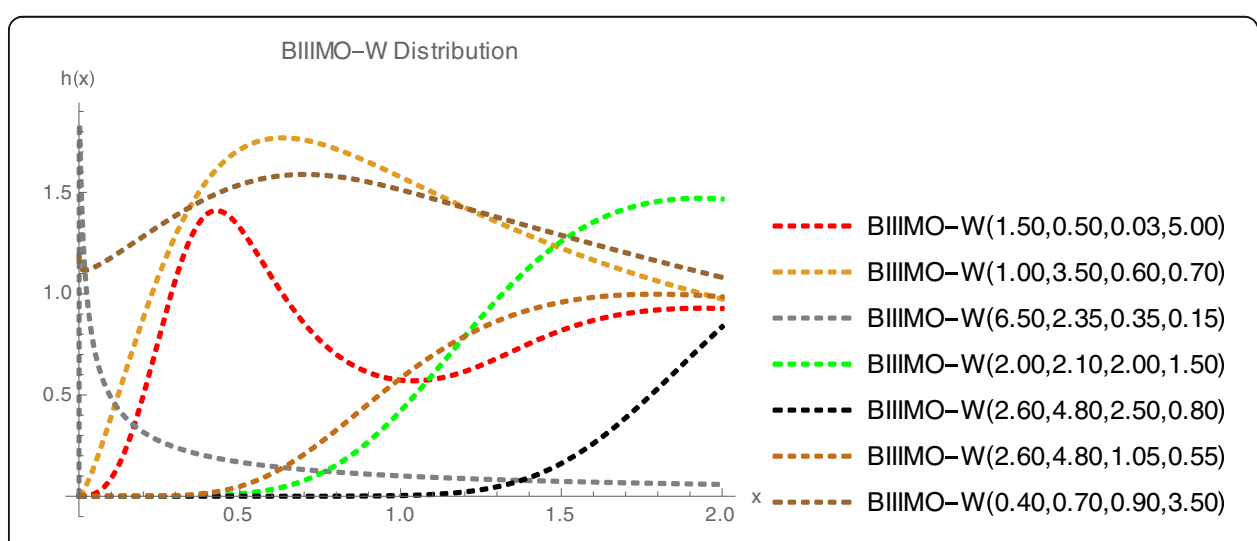

Fig. 2 Plots of hrf of BIIIMO-W distribution for selected parameter values 


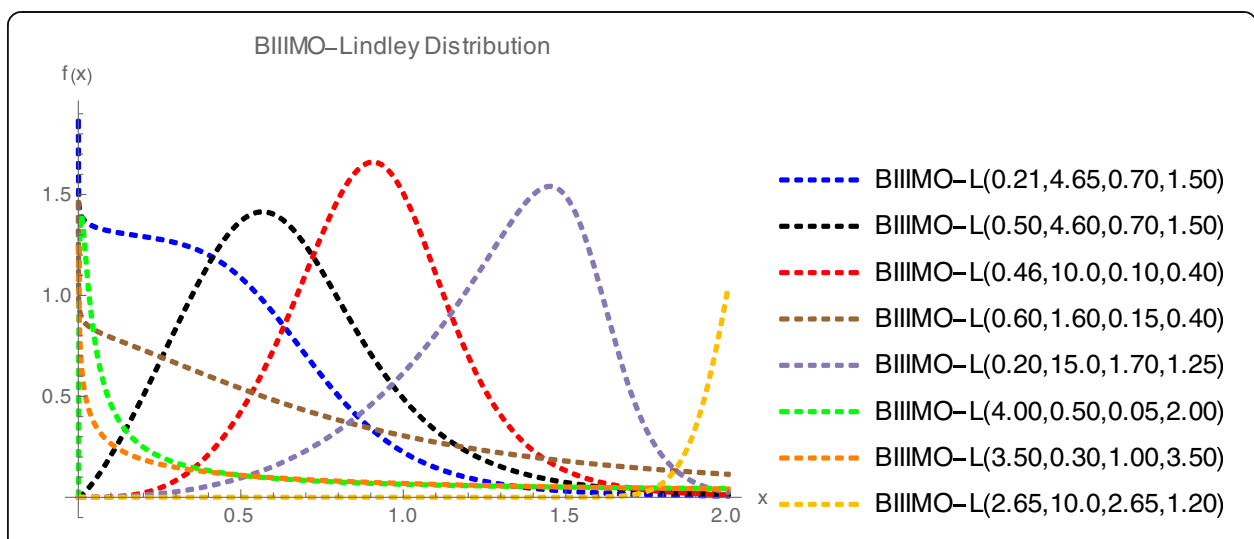

Fig. 3 Plots of pdf of BIIIMO-L distribution for selected parameter values

$$
\begin{aligned}
& p_{0}=1 \\
& p_{1}=-\frac{1}{2} i \beta \\
& p_{2}=\frac{1}{24}\left[3(i \beta)^{2}-5 i \beta\right], \\
& p_{3}=\frac{1}{48}\left[-(i \beta)^{3}+5(i \beta)^{2}-6 i \beta\right], \\
& p_{4}=\frac{1}{5760}\left[15(i \beta)^{4}-150(i \beta)^{3}+48(i \beta)^{2}-502 i \beta\right]
\end{aligned}
$$

etc., and

$$
q_{k}=q_{k}(i \beta)=\sum_{j=k}^{\infty}(-1)^{k+j}\left(\begin{array}{l}
i \beta \\
j
\end{array}\right)\left(\begin{array}{l}
j \\
k
\end{array}\right)
$$

Then, we arrive at

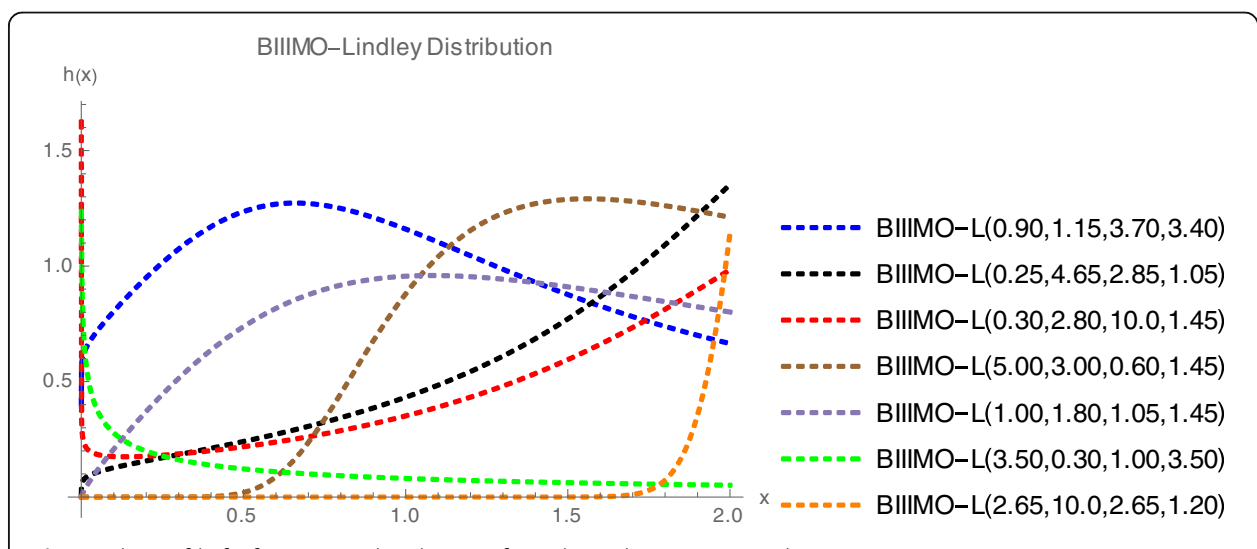

Fig. 4 Plots of hrf of BIIIMO-L distribution for selected parameter values 


$$
F(x)=1-\sum_{i \geq 1}^{\infty}(-1)^{i+1}\left(\begin{array}{l}
\alpha+i-1 \\
i
\end{array}\right) \frac{\sum_{k=0}^{\infty} p_{k}\left[1-\left(\frac{\lambda \bar{G}(x)}{1-\bar{\lambda} \bar{G}(x)}\right)\right]^{k}}{\sum_{k=0}^{\infty} q_{k}\left(1-\frac{\lambda \bar{G}(x)}{1-\bar{\lambda} \bar{G}(x)}\right)^{k}}
$$

Using

$$
\frac{\sum_{k=0}^{\infty} b_{k} x^{k}}{\sum_{k=0}^{\infty} a_{k} x^{k}}=\frac{1}{a_{0}} \sum_{k=0}^{\infty} c_{k} x^{k}
$$

where $c_{n}-\frac{1}{a_{0}} \sum_{k=1}^{n} c_{n-k} a_{k}-b_{n}=0$ (see Gradshteyn and Ryzhik 2014),

$$
\text { we obtain } F(x)=1-\frac{\sum_{i \geq 1}^{\infty}(-1)^{1+i}\left(\begin{array}{c}
\alpha+i-1 \\
{ }^{\alpha+i}
\end{array}\right)}{q_{0}} \sum_{k=0}^{\infty} c_{k} \underbrace{\left[-\left(\frac{\lambda \bar{G}(x)}{1-\bar{\lambda} \bar{G}(x)}\right)\right]}_{A} \text {. }
$$

By applying the power series to the quantity A,

$$
(1-z)^{k-1}=\sum_{h=0}^{\infty}(-1)^{h}\left(\begin{array}{l}
k-1 \\
h
\end{array}\right) z^{h}
$$

where $|z|<1$ and $\mathrm{b}$ is a real non-integer, we obtain

$$
F(x)=1-\sum_{i \geq 1}^{\infty}(-1)^{1+i}\left(\begin{array}{c}
\alpha+i-1 \\
i
\end{array}\right) \frac{1}{q_{0}}[c_{0}+\sum_{k=1}^{\infty} c_{k} \sum_{h=1}^{k}(-1)^{h} \lambda^{h}\left(\begin{array}{l}
k \\
h
\end{array}\right) \bar{G}(x)^{h} \underbrace{[1-(1-\lambda) \bar{G}(x)]}_{B}]^{-h} .
$$

For $0<\lambda \leq 1$, applying $(1-x)^{-n}=\sum_{\ell=0}^{\infty}\left(\begin{array}{l}n+\ell-1 \\ n-1\end{array}\right) x^{\ell}$ to the quantity $\mathrm{B}$, we arrive

$$
\begin{aligned}
& F(x)=1-\sum_{i \geq 1}^{\infty}(-1)^{1+i}\left(\begin{array}{c}
\alpha+i-1 \\
i
\end{array}\right) \frac{1}{q_{0}}\left[c_{0}+\sum_{k=1}^{\infty} c_{k} \sum_{h=1}^{k}(-1)^{h}\left(\begin{array}{c}
k \\
h
\end{array}\right) \lambda^{h} \sum_{j=0}^{\infty}\left(\begin{array}{c}
n+j-1 \\
n-1
\end{array}\right)(1-\lambda)^{j} \bar{G}(x)^{j+h}\right] \\
& =1+\left[\begin{array}{l}
\sum_{i \geq 1}^{\infty} \frac{c_{0}}{q_{0}}(-1)^{2+i}\left(\begin{array}{c}
\alpha+i-1 \\
i
\end{array}\right) \\
+\sum_{j=0}^{\infty} \sum_{h=1}^{k} \sum_{k=1}^{\infty} \sum_{i \geq 1}^{\infty} \frac{c_{k}}{q_{0}}(-1)^{2+h+i}(1-\lambda)^{j} \lambda^{h}\left(\begin{array}{c}
k \\
h
\end{array}\right)\left(\begin{array}{c}
n+j-1 \\
n-1
\end{array}\right)\left(\begin{array}{c}
\alpha+i-1 \\
i
\end{array}\right) \bar{G}(x)^{j+h}
\end{array}\right] \\
& F(x)=1+\sum_{i \geq 1}^{\infty} v_{i}+\sum_{j=0}^{\infty} \sum_{h=1}^{k} \sum_{k=1}^{\infty} v_{j, h} \bar{H}_{j+h}(x),
\end{aligned}
$$

where

$$
\begin{aligned}
& v_{j, h}=\sum_{i \geq 1}^{\infty} \frac{c_{k}}{q_{0}}(-1)^{2+h+i}(1-\lambda)^{j} \lambda^{h}\left(\begin{array}{l}
k \\
h
\end{array}\right)\left(\begin{array}{c}
n+j-1 \\
n-1
\end{array}\right)\left(\begin{array}{c}
\alpha+i-1 \\
i
\end{array}\right) \\
& v_{i}=\frac{c_{0}}{q_{0}}(-1)^{2+i}\left(\begin{array}{c}
\alpha+i-1 \\
i
\end{array}\right)
\end{aligned}
$$

and 


$$
\bar{H}_{\theta}(x)=\bar{G}(x)^{\theta}
$$

is the cdf of the Lehmann type II $\operatorname{Exp}(1-\mathrm{G})$ model with power $\theta>0$.

By differentiating (14) we get.

$$
f(x)=\sum_{j=0}^{\infty} \sum_{h=1}^{k} \sum_{k=1}^{\infty} v_{j, h} h_{j+h}(x),
$$

and

$$
h_{\theta}(x)=\theta g(x) \bar{G}(x)^{\theta-1}
$$

is the pdf of the Lehmann type II Exp (1-G) model with power $\theta>0$. Equations (14) and (15) reveal that the BIIIMO-G density can be written as linear combinations of the Lehmann type II $\operatorname{Exp}(1-\mathrm{G})$ density functions. So, all properties of the new family can be derived based on the Lehmann type II $\operatorname{Exp}(1-\mathrm{G})$ density.

\section{Moments}

Moments, incomplete moments, inequality measures and some other properties are theoretically derived in this section.

\section{Moments about origin}

The $r^{\text {th }}$ moment of $X$, say $\mu_{r}^{\prime}$, follows from (15) as

$$
\mu_{r}^{\prime}=E\left(X^{r}\right)=\sum_{j=0}^{\infty} \sum_{h=1}^{\mathrm{k}} \sum_{k=1}^{\infty} v_{j, h} E\left(Y_{m}^{r}\right)
$$

Henceforth, $Y_{m}$ denotes the Lehmann type II exp-(1-G) distribution with power parameter $m$.

The $n^{\text {th }}$ central moment of $X$, say $M_{n}$, is given by

$$
M_{n}=E\left(X-\mu_{1}^{\prime}\right)^{n}=\sum_{r=0}^{n} \sum_{j=0}^{\infty} \sum_{h=1}^{\mathrm{k}} \sum_{k=1}^{\infty} \mathrm{v}_{j, h}(-1)^{n-r}\left(\begin{array}{l}
n \\
r
\end{array}\right) \mu_{r}^{\prime(n-r)} E\left(Y_{m}^{r}\right)
$$

The cumulants $\left(\kappa_{n}\right)$ of $X$ follow recursively from

$$
\kappa_{n}=\mu_{n}^{\prime}-\sum_{r=0}^{n-1}\left(\begin{array}{c}
n-1 \\
r-1
\end{array}\right) \kappa_{r} \mu_{n-r}^{\prime}
$$

where $\kappa_{1}=\mu_{1}^{\prime}, \kappa_{2}=\mu_{2}^{\prime}-\mu_{1}^{\prime 2}, \kappa_{3}=\mu_{3}^{\prime}-3 \mu_{2}^{\prime} \mu_{1}^{\prime}+\mu_{1}^{\prime 3}$, etc. The skewness and kurtosis measures can be calculated from the ordinary moments using well-known relationships.

\section{Generating function}

The moment generating function (mgf) $M_{X}(t)=E\left(e^{t X}\right)$ of $X$ is given by

$$
M_{X}(t)=\sum_{j=0}^{\infty} \sum_{h=1}^{\mathrm{k}} \sum_{k=1}^{\infty} \mathrm{v}_{j, h} M_{m}(t)
$$

where $M_{m}(t)$ is the mgf of $Y_{m}$. Hence, $M_{X}(t)$ can be determined from Lehmann type II exp-(1-G) generating function. 


\section{Incomplete moments}

The $s^{\text {th }}$ incomplete moment, say $I_{s}(t)$, of $X$ can be expressed from (15) as

$$
I_{s}(t)=\int_{-\infty}^{t} x^{s} f(x) d x=\sum_{j=0}^{\infty} \sum_{h=1}^{\mathrm{k}} \sum_{k=1}^{\infty} \mathrm{v}_{j, h} \int_{-\infty}^{t} x^{s} h_{j+h}(x) d x
$$

A general formula for the first incomplete moment, $I_{1}(t)$, can be derived from the last equation (with $s=1$ ).

The first incomplete moment can be applied to construct Bonferroni and Lorenz curves defined for a given probability $\pi$ by $B(\pi)=I_{1}(q) /\left(\pi \mu_{1}^{\prime}\right)$ and $L(\pi)=I_{1}(q) / \mu_{1}^{\prime}$, respectively, where $\mu_{1}^{\prime}=E(X)$ and $q=Q(\pi)$ is the qf of $X$ at $\pi$. The mean deviations about the mean $\left[\delta_{1}=E\left(\left|X-\mu_{1}^{\prime}\right|\right)\right]$ and about the median $\left[\delta_{2}=E(|X-M|)\right]$ of $X$ are given by $\delta_{1}=2 \mu_{1}^{\prime} F\left(\mu_{1}^{\prime}\right)-2 I_{1}\left(\mu_{1}^{\prime}\right)$ and $\delta_{2}=\mu_{1}^{\prime}-2 I_{1}(M)$, respectively, where $\mu_{1}^{\prime}=E(X), M=$ Median $(X)=Q\left(\frac{1}{2}\right)$ is the median and $F\left(\mu_{1}^{\prime}\right)$ is easily calculated from (4).

Table 2 shows the numerical measures of the median, mean, standard deviation, skewness and Kurtosis of the BIIIMO-W distribution for selected parameter values to describe their effect on these measures.

Table 3 shows the numerical measures of the median, mean, standard deviation, skewness and Kurtosis of the BIIIMO-L distribution for selected parameter values to describe their effect on these measures.

\section{Reliability measures}

In this section, different reliability measures for the BIIIMO-G family are studied.

\section{Stress-strength reliability of BIIIMO-G family}

Let $X_{1} \sim B I I I M O-G\left(\alpha_{1}, \beta, \lambda, \psi\right), X_{2} \sim B I I I M O-G\left(\alpha_{2}, \beta, \lambda, \psi\right)$ and $X_{1}$ represents strength and $X_{2}$ represents stress. Then, the reliability of the component is:

$$
\begin{aligned}
\mathrm{R}= & \operatorname{Pr}\left(\mathrm{X}_{2}<X_{1}\right)=\int_{-\infty}^{\infty} \int_{-\infty}^{x_{1}} f\left(x_{1}, x_{2}\right) d x_{2} d x_{1}=\int_{0}^{\infty} f_{x_{1}}(x) F_{x_{2}}(x) d x, \\
R= & \int_{0}^{\infty} \frac{\alpha_{1} \beta g(x)}{\bar{G}(x)(1-\bar{\lambda} \bar{G}(x))}\left[-\log \left(\frac{\lambda \bar{G}(x)}{1-\bar{\lambda} \bar{G}(x)}\right)\right]^{-\beta-1}\left\{1+\left[-\log \left(\frac{\lambda \bar{G}(x)}{1-\bar{\lambda}(x)}\right)\right]^{-\beta}\right\}^{-\alpha_{1}-1} \\
& \left\{1+\left[-\log \left(\frac{\lambda \bar{G}(x)}{1-\bar{\lambda} \bar{G}(x)}\right)\right]^{-\beta}\right\}^{-\alpha_{2}} d x=\frac{\alpha_{1}}{\left(\alpha_{1}+\alpha_{2}\right)}
\end{aligned}
$$

Therefore $\mathbf{R}$ is independent of $\beta, \lambda$ and $\psi$.

\section{Multicomponent stress-strength reliability estimator $R_{s, \kappa}$ based on BIIIMO-G family}

Suppose a machine has at least "s" components working out of " $K$ " components. The strengths of all components of system are $\mathrm{X}_{1}, \mathrm{X}_{2}, \ldots \mathrm{X}_{\kappa}$ and stress $\mathrm{Y}$ is applied to the system. Both the strengths $\mathrm{X}_{1}, \mathrm{X}_{2}, \ldots \mathrm{X}_{\kappa}$ are i.i.d. and are independent of stress $\mathrm{Y} . \mathrm{F}$ 
Table 2 Median, mean, standard deviation, skewness and Kurtosis of the BIIIMO-W distribution

\begin{tabular}{llllll}
\hline Parameters $a, \beta, \lambda, b$ & Median & Mean & Standard Deviation & Skewness & Kurtosis \\
\hline $1,5,5,5$ & 1.1771 & 1.1780 & 0.0545 & 0.2111 & 4.9161 \\
$2,5,5,5$ & 1.2034 & 1.2079 & 0.04693 & 0.8716 & 5.9434 \\
$3,5,5,5$ & 1.2174 & 1.2232 & 0.0452 & 1.1058 & 6.6793 \\
$5,0.1,5,5$ & 1.7972 & 1.8793 & 0.9214 & 0.1150 & 1.9890 \\
$5,0.5,5,5$ & 1.9479 & 2.0645 & 0.6437 & 0.5428 & 2.4137 \\
$5,0.6,5,5$ & 1.8321 & 1.9746 & 0.6159 & 0.8302 & 2.9991 \\
$5,1,5,5$ & 1.5246 & 1.6301 & 0.3962 & 1.7014 & 6.7042 \\
$0.5,1.5,0.5,5$ & 0.8426 & 1.3586 & 1.1866 & 1.4186 & 3.2418 \\
$0.5,1.5,1.5,5$ & 0.9185 & 0.9232 & 0.2749 & 0.6282 & 5.9778 \\
$0.5,1.5,2.5,5$ & 0.9858 & 0.9805 & 0.2668 & 0.4229 & 5.6954 \\
$0.1,3.25,0.5,5$ & 0.5710 & 0.5575 & 0.2477 & -0.0171 & 2.9788 \\
$0.1,3.25,0.75,5$ & 0.6175 & 0.5985 & 0.2616 & -0.0403 & 3.5046 \\
$0.1,3.25,1,5$ & 0.6521 & 0.6282 & 0.2694 & -0.1980 & 2.2918 \\
$1,10,1,10$ & 1.0000 & 1.0001 & 0.0181 & 0.0954 & 4.2672 \\
$5,5,5,1$ & 2.8679 & 2.9859 & 0.5965 & 3.9302 & 127.836 \\
$5,5,5,3$ & 1.4208 & 1.4346 & 0.0866 & 1.4729 & 9.1049 \\
$5,5,5,5$ & 1.2346 & 1.2413 & 0.0443 & 1.3008 & 7.4790 \\
\hline
\end{tabular}

Table 3 Median, mean, standard deviation, skewness and Kurtosis of the BIIIMO-Lindley Distribution

\begin{tabular}{llllll}
\hline Parameters $a, \beta, \lambda, b$ & Median & Mean & Standard Deviation & Skewness & Kurtosis \\
\hline $1,1,1,1$ & 1.5675 & 5.3348 & 15.3851 & 8.2183 & 90.6069 \\
$2,1,1,1$ & 3.3663 & 9.5872 & 22.6103 & 6.4326 & 55.7313 \\
$3,1,1,1$ & 5.0424 & 13.5865 & 29.5541 & 5.8056 & 45.5406 \\
$4,1,1,1$ & 6.6589 & 17.6427 & 37.3102 & 5.5979 & 42.3776 \\
$5,1,1,1$ & 8.1887 & 19.9071 & 36.5353 & 4.6082 & 29.1756 \\
$1,10,1,1$ & 1.5826 & 1.6022 & 0.2585 & 0.8210 & 6.4705 \\
$0.65,10,5,1$ & 3.1084 & 3.1032 & 0.3673 & 0.0413 & 4.5913 \\
$0.65,10,5,0.15$ & 23.9115 & 23.8698 & 2.5003 & 0.0084 & 4.4424 \\
$0.5,10,3,0.10$ & 29.9001 & 29.7078 & 3.9877 & -0.1783 & 4.1985 \\
$0.15,20,0.5,0.95$ & 0.8711 & 0.8362 & 0.2341 & -0.5213 & 2.9375 \\
$0.15,20,0.5,0.5$ & 1.9486 & 1.8711 & 0.4854 & -0.5968 & 3.0644 \\
$0.5,20,0.5,0.5$ & 2.3013 & 2.2875 & 0.2677 & -0.2134 & 4.1862 \\
$0.1,9,0.5,0.5$ & 1.1787 & 1.2283 & 0.7764 & 0.4073 & 2.6804 \\
$0.1,9,0.35,0.5$ & 0.8983 & 0.9548 & 0.6292 & 0.5181 & 2.9726 \\
$0.1,10,1.1,0.4$ & 2.8248 & 2.7678 & 1.4233 & 0.0055 & 2.2482 \\
$0.1,10,1.3,0.4$ & 3.1227 & 3.0411 & 1.5263 & -0.0426 & 2.2369 \\
$0.1,10,2,0.4$ & 3.9758 & 3.8169 & 1.7934 & -0.1728 & 2.2366 \\
$0.1,10,2,1.8$ & 0.6578 & 0.6423 & 0.3405 & -0.0045 & 2.1782 \\
$0.1,10,2,1.9$ & 0.6160 & 0.6020 & 0.3203 & 0.0051 & 2.1889 \\
\hline & & & & &
\end{tabular}


and $\mathrm{G}$ are the cdf of $\mathrm{X}$ and $\mathrm{Y}$ respectively. The reliability of a machine is the probability that the machine functions properly.

Let $X \sim B I I I M O-G\left(\alpha_{1}, \beta, \lambda, \psi\right), Y \sim B I I I M O-G\left(\alpha_{2}, \beta, \lambda, \psi\right)$ with common parameters $\beta, \lambda, \psi$ and unknown shape parameters $\alpha_{1}$ and $\alpha_{2}$. The multicomponent stress- strength reliability for BIIIMO-G family is given by (Bhattacharyya and Johnson 1974).

$$
\begin{aligned}
& R_{s, \mathrm{~m}}=P(\text { strengths }>\text { stress })=P\left[\text { at least" } s^{\prime \prime} \text { of }\left(\mathrm{X}_{1}, \mathrm{X}_{2}, \ldots . \mathrm{X}_{K}\right) \text { exceed } Y\right] \\
& R_{s, K}=\sum_{l=s}^{K}\left(\begin{array}{l}
\kappa \\
\ell
\end{array}\right) \int_{0}^{\infty}[1-F(y)]^{l}[F(y)]^{K-l} d G(y) \\
& R_{s, K}=\sum_{\ell=s}^{K}\left(\begin{array}{l}
\kappa \\
\ell
\end{array}\right)\left[\begin{array}{l}
\left.\int_{0}^{\infty}\left(1-\left\{1+\left[-\log \left(\frac{\lambda \bar{G}(x)}{1-\bar{\lambda} \bar{G}(x)}\right)\right]^{-\beta}\right\}^{-\alpha_{1}}\right)^{\ell}\left(\left\{1+\left[-\log \left(\frac{\lambda \bar{G}(x)}{1-\bar{\lambda} \bar{G}(x)}\right)\right]^{-\beta}\right\}^{-\alpha_{1}}\right)^{(\kappa-\ell)} \times\right] d x \\
\frac{\alpha_{2} \beta g(x)}{\bar{G}(x)(1-\bar{\lambda} \bar{G}(x))}\left[-\log \left(\frac{\lambda \bar{G}(x)}{1-\bar{\lambda} \bar{G}(x)}\right)\right]^{-\beta-1}\left\{1+\left[-\log \left(\frac{\lambda \bar{G}(x)}{1-\bar{\lambda} \bar{G}(x)}\right)\right]^{-\beta}\right\}^{-\alpha_{2}-1}
\end{array}\right.
\end{aligned}
$$

Let $t=\left\{1+\left[-\log \left(\frac{\lambda \bar{G}(x)}{1-\bar{\lambda} \bar{G}(x)}\right)\right]^{-\beta}\right\}^{-\alpha_{2}}$, then we obtain

$$
\begin{aligned}
& R_{s, \kappa}=\sum_{\ell=s}^{\kappa}\left(\begin{array}{l}
\kappa \\
\ell
\end{array}\right) \int_{0}^{1}\left(1-t^{\nu}\right)^{\ell} t^{\nu(\kappa-\ell)} d t . \\
& \text { Let } z=t^{\nu}, t=z^{\frac{1}{\nu}}, d t=\frac{1}{v} z^{\frac{1}{\nu}-1} d z
\end{aligned}
$$

, then

$$
\begin{aligned}
R_{s, \kappa} & =\sum_{\ell=s}^{\kappa}\left(\begin{array}{l}
\kappa \\
\ell
\end{array}\right) \int_{0}^{1}\left(1-t^{\nu}\right)^{\ell} t^{\nu(\kappa-\ell)} d t, \\
R_{s, \kappa} & =\sum_{\ell=s}^{\kappa}\left(\begin{array}{l}
\kappa \\
\ell
\end{array}\right) \int_{0}^{1}(1-z)^{\ell} z^{(\kappa-\ell)} \frac{1}{v} z^{\frac{1}{\nu}-1} d z, \\
R_{s, \kappa} & =\frac{1}{v} \sum_{\ell=s}^{\kappa}\left(\begin{array}{c}
\kappa \\
\ell
\end{array}\right) B\left(1+\ell, \kappa-\ell+\frac{1}{v}\right) ., \\
R_{s, \kappa} & =\frac{1}{v} \sum_{\ell=s}^{\kappa} \frac{\kappa !}{(\kappa-\ell) !}\left[\prod_{j=0}^{\ell}\left(\kappa-j+\frac{1}{v}\right)\right]^{-1} \text { where } v=\frac{\alpha_{2}}{\alpha_{1}}
\end{aligned}
$$

The probability $R_{s, \kappa}$ in (24) is called multicomponent stress-strength model reliability.

\section{Characterizations}

In this section, BIIIMO-G family is characterized via: (i) conditional expectation; (ii) ratio of truncated moments and (iii) reverse hazard rate function.

\section{Characterization based on conditional expectation}

Here BIIIMO-G family is characterized via conditional expectation. 


\section{Proposition}

Let $X: \Omega \rightarrow(0, \infty)$ be a continuous random variable with cdf $F(x)$

$(0<F(x)<1$ for $x \geq 0)$, then for $\alpha>1$, X has cdf (4) if and only if

$$
E\left\{\left[-\log \left(\frac{\lambda \bar{G}(X)}{1-\bar{\lambda} \bar{G}(X)}\right)\right]^{-\beta} \mid X<t\right\}=\frac{1}{(\alpha-1)}\left\{1+\alpha\left[-\log \left(\frac{\lambda \bar{G}(t)}{1-\bar{\lambda} \bar{G}(t)}\right)\right]^{-\beta}\right\}, \text { for } t>0
$$

Proof. If pdf of $\mathrm{X}$ is (5), then

$$
\begin{gathered}
E\left\{\left[-\log \left(\frac{\lambda \bar{G}(X)}{1-\bar{\lambda} \bar{G}(X)}\right)\right]^{-\beta} \mid X<t\right\}=(F(t))^{-1} \int_{0}^{t}\left[-\log \left(\frac{\lambda \bar{G}(x)}{1-\bar{\lambda} \bar{G}(x)}\right)\right]^{-\beta} f(x) d x \\
=(F(t))^{-1} \int_{0}^{t}\left(\begin{array}{c}
{\left[-\log \left(\frac{\lambda \bar{G}(x)}{1-\bar{\lambda} \bar{G}(x)}\right)\right]^{-\beta} \alpha \beta \frac{g(x)}{\bar{G}(x)(1-\bar{\lambda} \bar{G}(x))} \times} \\
{\left[-\log \left(\frac{\lambda \bar{G}(x)}{1-\bar{\lambda} \bar{G}(x)}\right)\right]^{-\beta-1}\left\{1+\left[-\log \left(\frac{\lambda \bar{G}(x)}{1-\bar{\lambda} \bar{G}(x)}\right)\right]^{-\beta}\right\}^{-\alpha-1}}
\end{array}\right) d x
\end{gathered}
$$

Upon integration by parts and simplification, we obtain

$$
E\left\{\left[-\log \left(\frac{\lambda \bar{G}(X)}{1-\bar{\lambda} \bar{G}(X)}\right)\right]^{-\beta} \mid X<t\right\}=\frac{1}{(\alpha-1)}\left\{1+\alpha\left[-\log \left(\frac{\lambda \bar{G}(t)}{1-\bar{\lambda} \bar{G}(t)}\right)\right]^{-\beta}\right\}, \quad t>0 .
$$

Conversely, if (25) holds, then

$$
\int_{0}^{t}\left[-\log \left(\frac{\lambda \bar{G}(x)}{1-\bar{\lambda} \bar{G}(x)}\right)\right]^{-\beta} f(x) d x=\frac{F(t)}{(\alpha-1)}\left\{1+\alpha\left[-\log \left(\frac{\lambda \bar{G}(t)}{1-\bar{\lambda} \bar{G}(t)}\right)\right]^{-\beta}\right\}
$$

Differentiating (26) with respect to $t$, we obtain.

$$
\left[-\log \left(\frac{\lambda \bar{G}(t)}{1-\bar{\lambda} G(t)}\right)\right]^{-\beta} f(t)=\frac{f(t)}{(\alpha-1)}\left\{1+\alpha\left[-\log \left(\frac{\lambda \bar{G}(t)}{1-\bar{\lambda} \bar{G}(t)}\right)\right]^{-\beta}\right\}-\frac{F(t)}{(\alpha-1)}\left[\frac{\alpha \beta g(t)}{\bar{G}(x)(1-\bar{\lambda} \bar{G}(t))}\left[-\log \left(\frac{\lambda \bar{G}(t)}{1-\bar{\lambda} \bar{G}(t)}\right)\right]^{-\beta-1}\right]
$$

After simplification and integration we arrive at

$$
F(t)=\left\{1+\left[-\log \left(\frac{\lambda \bar{G}(t)}{1-\bar{\lambda} \bar{G}(t)}\right)\right]^{-\beta}\right\}^{-\alpha}, t \geq 0
$$

\section{Characterization of BIIIMO-G family through ratio of truncated moments}

Here BIIIMO-G family is characterized using Theorem G (Glänzel; 1987) on the basis of a simple relationship between two truncated moments of $\mathrm{X}$.

\section{Proposition}

Let $\mathrm{X}: \Omega \rightarrow \mathbb{R}$ be a continuous random variable.

$$
\text { Let } h_{1}(x)=\frac{1}{\alpha}\left\{1+\left[-\log \left(\frac{\lambda \bar{G}(x)}{1-\bar{\lambda} \bar{G}(x)}\right)\right]^{-\beta}\right\}^{\alpha+1}, x \in \mathbb{R}
$$

and 


$$
h_{2}(x)=\frac{2\left\{1+\left[-\log \left(\frac{\lambda \bar{G}(x)}{1-\bar{\lambda} \bar{G}(x)}\right)\right]^{-\beta}\right\}^{\alpha+1}}{\alpha\left[-\log \left(\frac{\lambda \bar{G}(x)}{1-\bar{\lambda} \bar{G}(x)}\right)\right]^{\beta}}, x \in \mathbb{R}
$$

The random variable $\mathrm{X}$ has pdf (5) if and only if that the function $p(x)$ (defined in theorem G) has the form $p(x)=\left[-\log \left(\frac{\lambda \bar{G}(x)}{1-\bar{\lambda} \bar{G}(x)}\right]^{\beta}, \quad x \in \mathbb{R}\right.$.

Proof. For random variable $\mathrm{X}$ with pdf (5),

$$
(1-F(x)) E\left(h_{1}(X) \mid X \geq x\right)=\left[-\log \left(\frac{\lambda \bar{G}(x)}{1-\bar{\lambda} \bar{G}(x)}\right)\right]^{-\beta}
$$

and

$$
\begin{aligned}
(1-F(x)) E\left(h_{2}(X) \mid X \geq x\right) & =\left[-\log \left(\frac{\lambda \bar{G}(x)}{1-\bar{\lambda} \bar{G}(x)}\right)\right]^{-2 \beta}, x \in \mathbb{R} . \\
\frac{E\left[h_{1}(x) \mid X \geq x\right]}{E\left[h_{2}(x) \mid X \geq x\right]} & =p(x)=\left[-\log \left(\frac{\lambda \bar{G}(x)}{1-\bar{\lambda} \bar{G}(x)}\right)\right]^{\beta}, x \in \mathbb{R},
\end{aligned}
$$

and

$$
p^{\prime}(x)=\frac{\beta g(x)}{\bar{G}(x)(1-\bar{\lambda} \bar{G}(x))}\left[-\log \left(\frac{\lambda \bar{G}(x)}{1-\bar{\lambda} \bar{G}(x)}\right]^{\beta-1}, x \in \mathbb{R} .\right.
$$

The differential equation

$$
s^{\prime}(x)=\frac{p^{\prime}(x) h_{2}(x)}{p(x) h_{2}(x)-h_{1}(x)}=\frac{2 \beta g(t)}{\bar{G}(x)(1-\bar{\lambda} \bar{G}(t))}\left[-\log \left(\frac{\lambda \bar{G}(x)}{1-\bar{\lambda} \bar{G}(x)}\right)\right]^{-1}
$$

has solution

$$
s(x)=\ln \left[-\log \left(\frac{\lambda \bar{G}(x)}{1-\bar{\lambda} \bar{G}(x)}\right)\right]^{2 \beta}, x \in \mathbb{R} .
$$

Therefore, in the light of theorem G, X has pdf (5).

\section{Corollary}

Let $\mathrm{X}: \Omega \rightarrow \mathbb{R}$ be a continuous random variable and let.

$$
h_{2}(x)=\frac{2\left\{1+\left[-\log \left(\frac{\bar{\lambda} \bar{G}(x)}{1-\bar{\lambda} G(x)}\right)^{-\beta}\right\}^{\alpha+1}\right.}{\alpha\left[-\log \left(\frac{\lambda \bar{G}(x)}{1-\bar{\lambda} \bar{G}(x)}\right)^{\beta}\right.}, x \in \mathbb{R} \text {. The pdf of } X \text { is (5) if and only if there exist }
$$

functions

$p(x)$ and $h_{1}(x)$ defined in Theorem $\mathrm{G}$, satisfying the differential equation

$$
\frac{p^{\prime}(x)}{p(x) h_{2}(x)-h_{1}(x)}=\frac{\alpha \beta g(x)}{\bar{G}(x)(1-\bar{\lambda} \bar{G}(x))}\left[-\log \left(\frac{\lambda \bar{G}(x)}{1-\bar{\lambda} \bar{G}(x)}\right)\right]^{\beta-1}\left\{1+\left[-\log \left(\frac{\lambda \bar{G}(x)}{1-\bar{\lambda} \bar{G}(x)}\right)\right]^{-\beta}\right\}^{-\alpha-1}
$$




\section{Remark}

The solution of (27) is

$$
p(x)=\left[-\log \left(\frac{\lambda \bar{G}(x)}{1-\bar{\lambda} \bar{G}(x)}\right)\right]^{2 \beta} \int\left[\begin{array}{r}
-h_{1}(x) \frac{\alpha \beta g(x)}{\bar{G}(x)(1-\bar{\lambda} \bar{G}(x))}\left[-\log \left(\frac{\lambda \bar{G}(x)}{1-\bar{\lambda} \bar{G}(x)}\right)\right]_{-\alpha-1}^{-\beta-1} \times \\
\left\{1+\left[-\log \left(\frac{\lambda \bar{G}(x)}{1-\bar{\lambda} \bar{G}(x)}\right)\right]^{-\beta}\right\}^{-\beta} d x
\end{array}\right]+D,
$$

where $\mathrm{D}$ is a constant.

\section{Characterization of BIIIMO-G family via reverse Hazard rate function}

Definition

Let $\mathrm{X}: \Omega \rightarrow(0, \infty)$ be a continuous random variable with $\operatorname{cdf} F(x)$ and pdf $f(x)$. The reverse hazard function, $r_{F}$, of a twice differentiable distribution function $\mathrm{F}$, satisfies the differential equation

$$
\frac{d}{d x}[\ln f(x)]=\frac{r_{F}^{\prime}(x)}{r_{F}(x)}+r_{F}(x)
$$

\section{Proposition}

Let $\mathrm{X}: \Omega \rightarrow(0, \infty)$ be continuous random variable. The pdf of $\mathrm{X}$ is (5) if and only if its reverse hazard function, $r_{F}$ satisfies the first order differential equation

$$
\begin{aligned}
& \mathrm{r}_{F}^{\prime}(\mathrm{x})\left(1+\left\{-\log \left[\frac{\lambda \bar{G}(x)}{1-\bar{\lambda} \bar{G}(x)}\right]\right\}^{-\beta}\right)-\frac{\beta g(x)\left[-\log \left(\frac{\lambda \bar{G}(x)}{1-\bar{\lambda} \bar{G}(x)}\right)\right]^{-\beta-1}}{\bar{G}(x)(1-\bar{\lambda} \bar{G}(x))} r_{F}(x) \\
& \quad=\alpha \beta \frac{d}{d x}\left\{\frac{g(x)\left[-\log \left(\frac{\lambda \bar{G}(x)}{1-\bar{\lambda} \bar{G}(x)}\right)\right]^{-\beta-1}}{\bar{G}(x)(1-\bar{\lambda} \bar{G}(x))}\right\} .
\end{aligned}
$$

Proof If

X has pdf (5), then (29) holds. Now if (29) holds, then

$$
\frac{d}{d x}\left[r_{F}(x)\left(1+\left\{-\log \left[\frac{\lambda \bar{G}(x)}{1-\bar{\lambda} \bar{G}(x)}\right]\right\}^{-\beta}\right)\right]=\alpha \beta \frac{d}{d x}\left\{\frac{g(x)}{\bar{G}(x)(1-\bar{\lambda} G(x))}\left[-\log \left(\frac{\lambda \bar{G}(x)}{1-\bar{\lambda} \bar{G}(x)}\right)\right]^{-\beta-1}\right\},
$$

or

$$
r_{F}(x)=\frac{\alpha \beta g(x)}{\bar{G}(x)(1-\bar{\lambda} \bar{G}(x))}\left[-\log \left(\frac{\lambda \bar{G}(x)}{1-\bar{\lambda} \bar{G}(x)}\right)\right]^{-\beta-1}\left\{1+\left[-\log \left(\frac{\lambda \bar{G}(x)}{1-\bar{\lambda} \bar{G}(x)}\right)\right]^{-\beta}, x \in \mathbb{R}\right.
$$

which is the reverse hazard function of the BIIIMO-G Family.

\section{Maximum likelihood estimation}

In this section, parameter estimates are derived using maximum likelihood method. The log-likelihood function for the vector of parameters $\Phi=\left(\alpha, \beta, \lambda, \psi^{T}\right)$ of BIIIMO-G family is 


$$
\begin{aligned}
\ell(\Phi)=\operatorname{lnL}(\Phi) \\
=\left(\begin{array}{l}
n \ln (\alpha)+n \ln (\beta)+\sum_{i=1}^{n} \ln g\left(x_{i} ; \psi\right)-\sum_{i=1}^{n} \ln \bar{G}\left(x_{i} ; \psi\right)- \\
\sum_{i=1}^{n} \ln \left(1-\bar{\lambda} \bar{G}\left(x_{i} ; \psi\right)\right)-(\beta+1) \sum_{i=1}^{n} \ln \left[-\log \left(\frac{\lambda \bar{G}\left(x_{i} ; \psi\right)}{1-\bar{\lambda} \bar{G}\left(x_{i} ; \psi\right)}\right)\right]- \\
(\alpha+1) \sum_{i=1}^{n} \ln \left\{1+\left[-\log \left(\frac{\lambda \bar{G}\left(x_{i} ; \psi\right)}{1-\bar{\lambda} \bar{G}\left(x_{i} ; \psi\right)}\right)\right]^{-\beta}\right\}
\end{array}\right)
\end{aligned}
$$

In order to compute the estimates of the parameters $\alpha, \beta, \lambda, \psi^{\tau}$, the following nonlinear equations must be solved simultaneously:

$$
\begin{aligned}
& \frac{\delta}{\delta \alpha} \ell(\Phi)=\frac{n}{\alpha}-\sum_{i=1}^{n} \ln \left\{1+\left[-\log \left(\frac{\lambda \bar{G}\left(x_{i} ; \psi\right)}{1-\bar{\lambda} \bar{G}\left(x_{i} ; \psi\right)}\right)\right]^{-\beta}\right\}=0 \\
& \frac{\delta}{\delta \beta} \ell(\Phi)=\frac{n}{\beta}-\sum_{i=1}^{n} \ln \left[-\log \left(\frac{\lambda \bar{G}\left(x_{i} ; \psi\right)}{1-\bar{\lambda} \bar{G}\left(x_{i} ; \psi\right)}\right)\right]+(\alpha+1) \sum_{i=1}^{n} \ln \left[-\log \left(\frac{\lambda \bar{G}\left(x_{i} ; \psi\right)}{1-\bar{\lambda} G\left(x_{i} ; \psi\right)}\right)\right]\left\{\left[-\log \left(\frac{\lambda \bar{G}\left(x_{i} ; \psi\right)}{1-\bar{\lambda} \bar{G}\left(x_{i} ; \psi\right)}\right)\right]^{\beta}+1\right\}^{-1}, \\
& \frac{\delta}{\delta \psi} \ell(\Phi)=\left(\begin{array}{l}
\sum_{i=1}^{n} \frac{g^{\prime}\left(x_{i} ; \psi\right)}{g\left(x_{i} ; \psi\right)}+\sum_{i=1}^{n} \frac{g^{\prime}\left(x_{i} ; \psi\right)}{\bar{G}\left(x_{i} ; \psi\right)}-\sum_{i=1}^{n} \frac{\bar{\lambda}^{\prime}\left(x_{i} ; \psi\right)}{\left[1-\bar{\lambda} \bar{G}\left(x_{i} ; \psi\right)\right]} \\
-(\beta+1) \sum_{i=1}^{n} \frac{g^{\prime}\left(x_{i} ; \psi\right)\left[-\log \left(\frac{\lambda \bar{G}\left(x_{i} ; \psi\right)}{1-\bar{\lambda}\left(x_{i} ; \psi\right)}\right)\right]^{-1}}{\left(\bar{G}\left(x_{i} ; \psi\right)-\bar{\lambda} \bar{G}^{2}\left(x_{i} ; \psi\right)\right)} \\
-(\alpha+1) \beta \sum_{i=1}^{n}\left\{1+\left[-\log \left(\frac{\lambda \bar{G}\left(x_{i} ; \psi\right)}{1-\bar{\lambda} \bar{G}\left(x_{i} ; \psi\right)}\right)\right]^{\beta}\right\} \frac{g^{\prime}\left(x_{i} ; \psi\right)\left[-\log \left(\frac{\lambda \bar{G}\left(x_{i} ; \psi\right)}{1-\bar{G}\left(x_{i} \psi\right)}\right)\right]^{\beta-1}}{\left(\bar{G}\left(x_{i} ; \psi\right)-\bar{\lambda} \bar{G}\left(x_{i} ; \psi\right)\right)}
\end{array}\right)=0 .
\end{aligned}
$$

where $g^{\prime}\left(x_{i} ; \psi\right)=\frac{\delta}{\delta \psi} g\left(x_{i} ; \psi\right)$.

\section{Simulation studies}

In this Section, we perform the simulation study to see the performance of MLE's of BIIIMO-L distribution. The random number generation is obtained with inverse of its cdf. The MLEs, say $\left(\hat{\alpha}_{i}, \hat{\beta}_{i}, \hat{\lambda}_{i}, \hat{b}_{i}\right)$ for $\mathrm{i}=1,2, \ldots, \mathrm{N}$, have been obtained by CG routine in $\mathrm{R}$ programme.

The simulation study is based on graphical results. We generate $N=1000$ samples of sizes $n=20,30, \ldots, 1000$ from BIIIMOL distribution and get true values of $\alpha=1.5, \beta=$ 2.55, $\lambda=0.095$ and $b=5$ for this simulation study. We also calculate the mean, standard deviations (sd), bias and mean square error (MSE) of the MLEs. The bias and MSE are calculated by (for $h=\alpha, \beta, \lambda, b)$

$$
\text { Bias }_{h}=\frac{1}{N} \sum_{i=1}^{N}\left(\hat{h}_{i}-h\right)
$$

and

$$
M S E_{h}=\frac{1}{N} \sum_{i=1}^{N}\left(\hat{h}_{i}-h\right)^{2} .
$$

The results are given by Fig. 5 . Figure 5 reveals that the empirical means tend to the true parameter values and that the sds, biases and MSEs decrease when the sample size 
increases as expected. These results are in agreement with first-order asymptotic theory.

\section{Applications}

The BIIIMO-W and BIIIMO-L distributions are compared with sub- and competing models. Different goodness fit measures such Cramer-von Mises (W*), Anderson Darling (A*), Kolmogorov- Smirnov statistics with $p$-values [K-S(p-values], Akaike information criterion (AIC), consistent Akaike information criterion (CAIC), Bayesian information criterion (BIC), Hannan-Quinn information criterion (HQIC) and likelihood ratio statistics are computed for survival times of leukemia patients and bladder cancer patients using R-Packages(AdequacyModel and zipfR). In first application, we compare BIIIMO-W distribution with Weibull Marshall Olkin-Weibull (WMO-W), odd Burr III Weibull (OBIII-W), Kumaraswamy Weibull (Kum.-W), beta Weibull (Beta-W), generalized gamma Weibull, Weibull and Burr III (BIII) distributions. In second application, we compare BIIIMO-Lindley (BIIIMO-L) distribution with odd Burr III Lindley (OBIII-L), Mc-Donald- Lindley (Mc-L), Kumaraswamy Lindley (Kum.-L), beta Lindley (Beta-L), Weibull power Lindley (W-PL), Kumaraswamy power Lindley (Kum.- PL), Lindley and Burr III (BIII) distributions.

The better fit corresponds to smaller $\mathrm{W}^{*}, \mathrm{~A}^{*}, \mathrm{~K}-\mathrm{S},-2 \widehat{\ell}$, AIC, CAIC, BIC and HQIC value. The maximum likelihood estimates (MLEs) of unknown parameters and values of goodness of fit measures are computed for BIIIMO-W, BIIIMO-L distributions and their sub-models and competing models. The MLEs, their standard errors (in parentheses) and goodness-of-fit statistics like $\mathrm{W}^{*}, \mathrm{~A}^{*}, \mathrm{~K}-\mathrm{S}$ (p-value) are given in Tables 4 and 5. Tables 6 and 7 displays goodness-of-fit values.

\section{Data set I}

The survival times (days) of 40 patients suffering from leukemia (Abouammoh et al. 1994) are: $115,181,255,418,441,461,516,739,743,789,807,865,924,983$, 1024,1062 , 1063,1165, 1191, 1222,1251,1277, 1290,1357,1369, 1408,1455, 1478, 1222,1549, 1578, 1578, 1599, 1603, 1605, 1696, 1735, 1799, 1815,1852.

The BIIIMO-W distribution is best fitted than sub-models and competing models because the values of all criteria are smaller for BIIIMO-W distribution.

We can also perceive that the BIIIMO-W distribution is best fitted model than other sub-models and competing models because BIIIMO-W distribution offers the closer fit to empirical data (Fig. 6).

\section{Data set II}

The survival times of 128 bladder cancer patients (Lee and Wang 2003) are 0.08, $2.09,3.48,4.87,6.94,8.66,13.11,23.63,0.20,2.23,3.52,4.98,6.97,9.02,13.29$, $0.40,2.26,3.57,5.06,7.09,9.22,13.80,25.74,0.50,2.46,3.64,5.09,7.26,9.47$, $14.24,25.82,0.51,2.54,3.70,5.17,7.28,9.74,14.76,26.31,0.81,2.62,3.82,5.32$, $7.32,10.06,14.77,32.15,2.64,3.88,5.32,7.39,10.34,14.83,34.26,0.90,2.69$, $4.18,5.34,7.59,10.66,15.96,36.66,1.05,2.69,4.23,5.41,7.62,10.75,16.62$, $43.01,1.19,2.75,4.26,5.41,7.63,17.12,46.12,1.26,2.83,4.33,5.49,7.66,11.25$, $17.14,79.05,1.35,2.87,5.62,7.87,11.64,17.36,1.40,3.02,4.34,5.71,7.93,11.79$, 


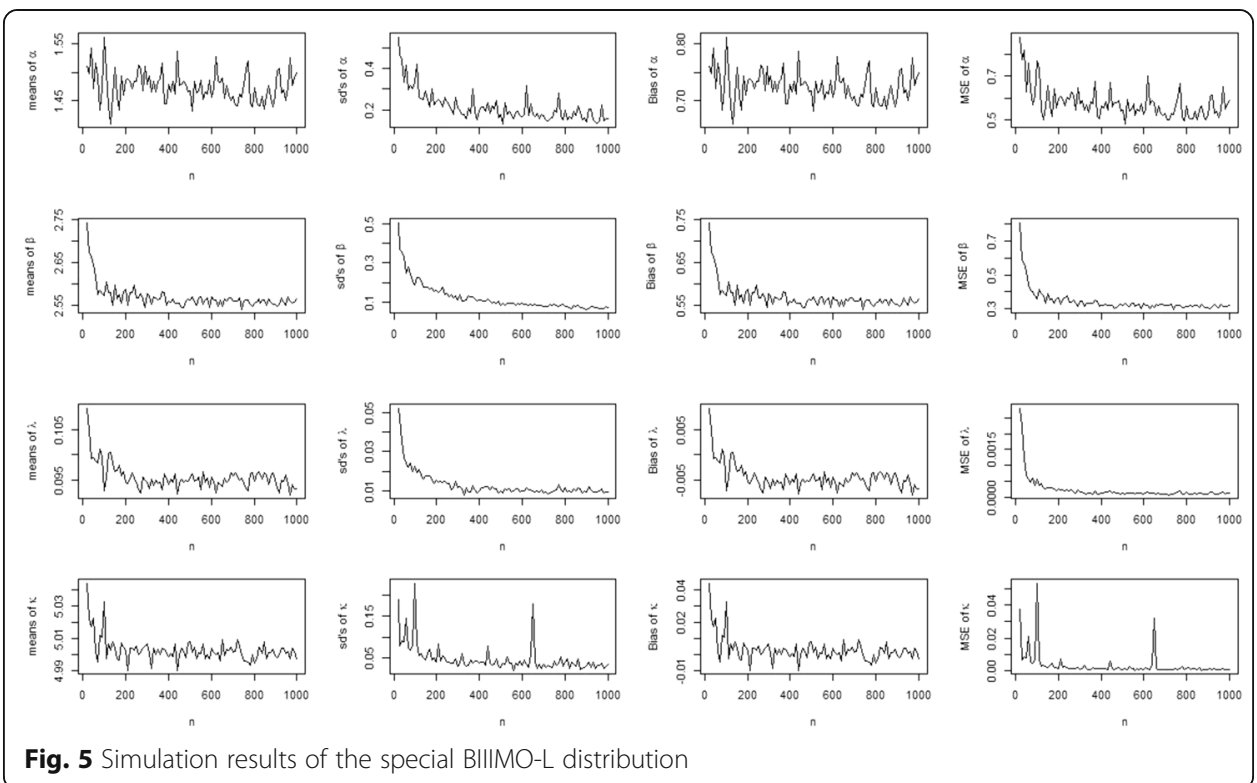

$18.10,1.46,4.40,5.85,8.26,11.98,19.13,1.76,3.25,4.50,6.25,8.37,12.02,2.02$, $3.31,4.51,6.54,8.53,12.03,20.28,2.02,3.36,6.76,12.07,21.73,2.07,3.36,6.93$, $8.65,12.63,22.69$

The BIIIMO-L distribution is best fitted than sub-models and competing models because the values of all criteria are smaller for BIIIMO-L distribution.

We can also perceive that the BIIIMO-L distribution is best fitted model than other sub-models and competing models because BIIIMO-L distribution offers the closer fit to empirical data (Fig. 7).

Table 4 MLEs, their standard errors (in parentheses) and Goodness-of-fit statistics for data

\begin{tabular}{|c|c|c|c|c|c|c|c|c|}
\hline Model & a & $\beta$ & $\lambda$ & $a$ & $b$ & $\mathrm{~W}^{*}$ & $A^{*}$ & K-S ( $p$-value) \\
\hline BIIIMO-W & $\begin{array}{l}0.0778 \\
(0.0535)\end{array}$ & $\begin{array}{l}61.6872 \\
(50.1271)\end{array}$ & $\begin{array}{l}15.3084 \\
(8.1416)\end{array}$ & - & $\begin{array}{l}0.1597 \\
(0.0214)\end{array}$ & 0.0223 & 0.1689 & $\begin{array}{l}0.0657 \\
(0.9952)\end{array}$ \\
\hline WMO-W & - & $\begin{array}{l}1.4827 \\
(0.8886)\end{array}$ & $\begin{array}{l}19.8894 \\
(50.0788)\end{array}$ & $\begin{array}{l}0.0100 \\
(0.0090)\end{array}$ & $\begin{array}{l}0.8196 \\
(0.0880)\end{array}$ & 0.1010 & 0.6747 & $\begin{array}{l}0.0917 \\
(0.8897)\end{array}$ \\
\hline OBIII-W & $\begin{array}{l}8.3649 \\
(1.9540)\end{array}$ & $\begin{array}{l}0.3518 \\
(0.2383)\end{array}$ & - & $\begin{array}{l}0.0071 \\
(0.0027)\end{array}$ & $\begin{array}{l}0.9864 \\
(0.1077)\end{array}$ & 0.2146 & 1.3316 & $\begin{array}{l}0.1349 \\
(0.4609)\end{array}$ \\
\hline Kum.-W & $\begin{array}{l}5.3277 \\
(1.4325)\end{array}$ & $\begin{array}{l}1.0881 \\
(0.6411)\end{array}$ & - & $\begin{array}{l}0.0071 \\
(0.0028)\end{array}$ & $\begin{array}{l}0.8185 \\
(0.0713)\end{array}$ & 0.4722 & 2.7197 & $\begin{array}{l}0.8567 \\
(<2.2 \mathrm{e}-16)\end{array}$ \\
\hline Beta-W & $\begin{array}{l}686.6493 \\
(275.8812)\end{array}$ & $\begin{array}{l}117.5352 \\
(76.0463)\end{array}$ & - & $\begin{array}{l}11.0073 \\
(15.0129)\end{array}$ & $\begin{array}{l}0.0705 \\
(0.0154)\end{array}$ & 0.4182 & 2.4380 & $\begin{array}{l}0.1769 \\
(0.1637)\end{array}$ \\
\hline GG-W & $\begin{array}{l}20.12230 \\
(2.7548)\end{array}$ & $\begin{array}{l}0.9595 \\
(0.2615)\end{array}$ & - & $\begin{array}{l}2.4800 \\
(0.1540)\end{array}$ & $\begin{array}{l}0.3978 \\
(0.1058)\end{array}$ & 0.3531 & 2.0916 & $\begin{array}{l}0.1749 \\
(0.1729)\end{array}$ \\
\hline Weibull & - & - & - & $\begin{array}{l}0.0743 \\
(0.3914)\end{array}$ & $\begin{array}{l}1.9547 \\
(10.3026)\end{array}$ & 0.4920 & 2.8243 & $\begin{array}{l}0.5085 \\
(2.069 \mathrm{e}-09)\end{array}$ \\
\hline BIII & - & - & - & $\begin{array}{l}1000.0297 \\
(545.5985)\end{array}$ & $\begin{array}{l}1.0548 \\
(0.0879)\end{array}$ & 0.6829 & 3.8003 & $\begin{array}{l}0.3005 \\
(0.001459)\end{array}$ \\
\hline
\end{tabular}


Table 5 MLEs, their standard errors (in parentheses) and Goodness-of-fit statistics for data set II

\begin{tabular}{|c|c|c|c|c|c|c|c|c|c|}
\hline Model & $a$ & $\beta$ & $\lambda$ & $a$ & $b$ & c & $\mathrm{W}^{*}$ & $A^{*}$ & K-S ( $p$-value) \\
\hline $\begin{array}{l}\text { BlIIMO- } \\
\mathrm{L}\end{array}$ & $\begin{array}{l}0.5122 \\
(0.2016)\end{array}$ & $\begin{array}{l}1.8631 \\
(0.5179)\end{array}$ & $\begin{array}{l}0.5600 \\
(0.6845)\end{array}$ & - & $\begin{array}{l}0.1530 \\
(0.1159)\end{array}$ & - & 0.0168 & 0.1031 & $\begin{array}{l}0.0307 \\
(0.9997)\end{array}$ \\
\hline OBIII-L & $\begin{array}{l}0.7847 \\
(0.2147)\end{array}$ & $\begin{array}{l}0.9553 \\
(0.1586)\end{array}$ & - & - & $\begin{array}{l}0.1723 \\
(0.0330)\end{array}$ & - & 0.1903 & 1.1378 & $\begin{array}{l}0.0934 \\
(0.2145)\end{array}$ \\
\hline$M C-L$ & $\begin{array}{l}1.2106 \\
(0.7307)\end{array}$ & $\begin{array}{l}0.2846 \\
(0.0286)\end{array}$ & - & - & $\begin{array}{l}0.4949 \\
(0.0028)\end{array}$ & $\begin{array}{l}0.7145 \\
(0.5179)\end{array}$ & 0.1444 & 0.8629 & $\begin{array}{l}0.0884 \\
(0.2704)\end{array}$ \\
\hline Kum.-L & $\begin{array}{l}0.9611 \\
(0.1671)\end{array}$ & $\begin{array}{l}0.2825 \\
(0.0276)\end{array}$ & - & - & $\begin{array}{l}0.5015 \\
(0.0028)\end{array}$ & - & 0.1344 & 0.8039 & $\begin{array}{l}0.0898 \\
(0.2529)\end{array}$ \\
\hline Beta-L & $\begin{array}{l}0.9248 \\
(0.1301)\end{array}$ & $\begin{array}{l}0.2813 \\
(0.0283)\end{array}$ & - & - & $\begin{array}{l}0.4949 \\
(0.0037)\end{array}$ & - & 0.1399 & 0.836 & $\begin{array}{l}0.0894 \\
(0.2576)\end{array}$ \\
\hline W-PL & $\begin{array}{l}0.0215 \\
(0.0285)\end{array}$ & $\begin{array}{l}13.54214 \\
(12.6100)\end{array}$ & - & $\begin{array}{l}0.0466 \\
(0.0402)\end{array}$ & $\begin{array}{l}1.1848 \\
(0.0901)\end{array}$ & - & 0.1482 & 0.8851 & $\begin{array}{l}0.0735 \\
(0.4932)\end{array}$ \\
\hline $\begin{array}{l}\text { Kum- } \\
\text { PL }\end{array}$ & $\begin{array}{l}3.1406 \\
(2.9576)\end{array}$ & $\begin{array}{l}1.4481 \\
(3.0649)\end{array}$ & - & $\begin{array}{l}0.5045 \\
(0.3671)\end{array}$ & $\begin{array}{l}0.8239 \\
(0.3653)\end{array}$ & - & 0.0389 & 0.2541 & $\begin{array}{l}0.043 \\
(0.9719)\end{array}$ \\
\hline Lindley & - & - & - & - & $\begin{array}{l}0.1960 \\
(0.0123)\end{array}$ & - & 0.1717 & 1.0257 & $\begin{array}{l}0.1164 \\
(0.06235)\end{array}$ \\
\hline BIII & $\begin{array}{l}4.2070 \\
(0.4054)\end{array}$ & $\begin{array}{l}1.0333 \\
(0.0604)\end{array}$ & - & - & - & - & 0.3856 & 2.4543 & $\begin{array}{l}0.1017 \\
(0.1413)\end{array}$ \\
\hline
\end{tabular}

Table 6 Goodness-of-fit statistics for data set I

\begin{tabular}{llllll}
\hline Model & $-2 \hat{\ell}$ & AIC & CAIC & BIC & HQIC \\
\hline BIIIMO-W & 598.7174 & 606.7174 & 607.8603 & 613.4729 & 609.16 \\
WMO-W & 606.9106 & 614.9106 & 616.0534 & 621.6661 & 617.3531 \\
OBIII-W & 614.6548 & 622.6549 & 623.7977 & 629.4104 & 625.0974 \\
Kum.W & 622.6082 & 630.6082 & 631.7511 & 637.3637 & 633.0508 \\
Beta-W & 627.9062 & 635.9063 & 637.0491 & 642.6618 & 638.3489 \\
GG-W & 623.4344 & 631.4344 & 632.5772 & 638.1899 & 633.8769 \\
Weibull & 785.6506 & 789.6507 & 789.975 & 793.0284 & 790.872 \\
BIII & 652.7072 & 656.7072 & 657.0316 & 660.085 & 657.9285 \\
\hline
\end{tabular}

Table 7 Goodness-of-fit statistics for data set ॥

\begin{tabular}{llllll}
\hline Model & $-2 \hat{\ell}$ & AIC & CAIC & BIC & HQIC \\
\hline BIIIMO-L & 409.7209 & 827.4419 & 827.7671 & 838.85 & 832.0771 \\
OBIII-L & 416.2479 & 838.4958 & 838.6893 & 847.0519 & 841.9722 \\
Kum.-L & 414.0998 & 834.1995 & 834.3931 & 842.7556 & 837.6759 \\
Mc-L & 414.0916 & 836.1833 & 836.5085 & 847.5914 & 840.8184 \\
Beta-L & 414.1904 & 834.3807 & 834.5743 & 842.9368 & 837.8571 \\
WPL & 414.9072 & 837.8144 & 838.1397 & 849.2226 & 842.4496 \\
Kum.-PL & 410.4152 & 828.8304 & 829.1556 & 840.2386 & 833.4656 \\
Lindley & 419.5299 & 841.0598 & 841.0916 & 843.9118 & 842.2186 \\
BIII & 426.6864 & 857.3729 & 857.4689 & 863.0769 & 859.6905 \\
\hline
\end{tabular}



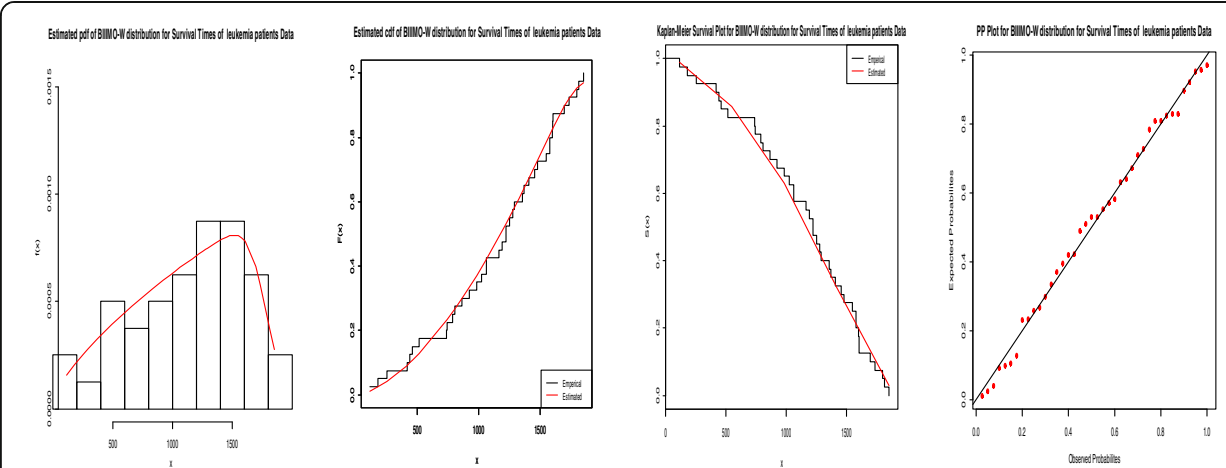

Fig. 6 Fitted pdf, cdf, survival and pp. plots of the BIIIMO-W distribution for survival times data

\section{Concluding remarks}

We have developed the BIIIMO-G family via the T-X family technique. We have studied properties such as sub-models; descriptive measures based on the quantiles, moments, inequality measures, stress-strength reliability and multicomponent stress-strength reliability model. The BIIIMO-G family is characterized via different techniques. The MLEs for the BIIIMO-G family have been computed. A simulation studies is performed to illustrate the performance of the maximum likelihood estimates (MLEs). Applications of the BIIIMO-G model to real data sets (survival times of leukemia patients and bladder cancer patients data) are presented to show the significance and flexibility of the BIIIMO-G family. Goodness of fit shows that the BIIIMO-G family is a better fit. We have demonstrated that the BIIIMO-G family is empirically better for lifetime applications.

\section{Abbreviations}

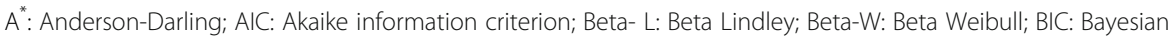
information criterion; BIII: Burr III; BIIIMO-G: Burr III Marshall Olkin-G; BIIIMO-L: Burr III Marshall Olkin-Lindley; BIIIMOW: Burr III Marshall Olkin-Weibull; CAIC: Consistent Akaike Information Criterion; cdf: Cumulative distribution function; GG-W: Generalized gamma Weibull; GIW-MO: Generalized inverse Weibull-Marshall Olkin; HQIC: Hannan-Quinn information criterion; hrf: Hazard rate function; i.i.d: Independent and identically distributed; KS: Kolmogorov-Smirnov; Kum.-L: Kumaraswamy Lindley; Kum.-PL: Kumaraswamy power Lindley; Kum.-W: Kumaraswamy Weibull; Mc-L: McDonald- Lindley; MLEs: Maximum likelihood estimators; MO-G: Marshall and Olkin; MSE: Mean square error; OBIII-L: Odd Burr III Lindley; OBIII-W: Odd Burr III Weibull; pdf: Probability density function; pp: Probability-Probability; rv: Random variable; sd: Standard deviations; W*: Cramer-von Mises; WMO-W: Weibull Marshall Olkin-Weibull; W-PL: Weibull power Lindley
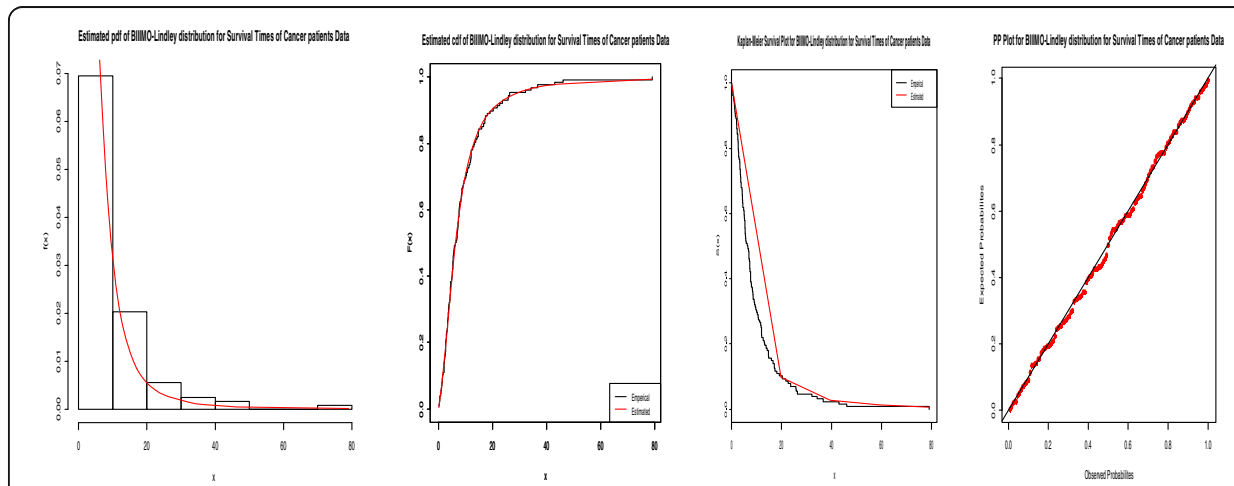

Fig. 7 Fitted pdf, cdf, survival and pp. plots of the BllIMO-Lindley distribution for survival times data 


\section{Acknowledgments}

The authors are grateful to the Editor-in-Chief, the Associate Editor and anonymous reviewers for their constructive comments and suggestions which led to remarkable improvement of the paper.

\section{Authors' contributions}

FAB proposed the BllIMO-G family of distributions and wrote the initial draft of the manuscript. The authors, viz. FAB, GGH, MCK, GMC, HMY and MA with the consultation of each other finalized this work. All authors read and approved the final manuscript.

\section{Funding}

GGH (co-author of the manuscript) is an Associate Editor of JSDA, 100\% discount on Article Processing Charge (APC) for the article).

\section{Availability of data and materials}

The BIIIMO-G family of distributions is derived from the idea of T-X family of distributions (Alzaatreh et al. 2013). All the data sets such as survival times of leukemia patients (Abouammoh et al. 1994) and survival times of bladder cancer patients (Lee and Wang 2003) are already available online. All the data and material about the article titled: BIIIMO-G family of distributions will also be available as per JSDA policy.

\section{Competing interests}

The authors declare that they have no competing interests.

\section{Author details}

${ }^{1}$ National College of Business Administration and Economics, Lahore, Pakistan. ${ }^{2}$ Marquette University, Milwaukee, WI 53201-1881, USA. ${ }^{3}$ Department of Measurement and Evaluation, Artvin Çoruh University, Artvin, Turkey.

${ }^{4}$ DepartamentodeEstatística, UniversidadeFederaldePernambuco, Recife, PE, Brazil. ${ }^{5}$ Department of Statistics Mathematics and Insurance, Benha University, Benha, Egypt.

Received: 19 March 2019 Accepted: 25 July 2019

Published online: 23 August 2019

\section{References}

Abouammoh, A.M., Abdulghani, S.A., Qamber, L.S.: On partialordering and testing of new better than renewal used class. Reliab. Eng. Syst. Saf. 25, 207-217 (1994)

Afify, A.Z., Cordeiro, G.M., Yousof, H.M., Saboor, A., Ortega, E.M.M.: The Marshall-Olkin additive Weibull distribution with variable shapes for the hazard rate. Hacet. J. Math. Stat. 47, 365-381 (2018)

Alizadeh, M., Cordeiro, G. M., De Brito, E., Demétrio, C. G. B. The beta Marshall-Olkin family of distributions. Journal of Statistical Distributions and Applications, 2(1), 4(2015).

Alzaatreh, A., Lee, C., Famoye, F.: A new method for generating families of continuous distributions. Metron. 71, 63-79 (2013)

Bhattacharyya, G.K., Johnson, R.A.: Estimation of reliability in a multicomponent stress-strength model. J. Am. Stat. Assoc 69(348), 966-970 (1974)

Cordeiro, G.M., Lemonte, A.J.: On the Marshall-Olkin extended Weibull distribution. Stat. Pap. 54, 333-353 (2013)

Ghitany, M.E., Al-Awadhi, F.A., Alkhalfan, L.A.: MarshallOlkin extended Lomax distribution and its application to censored data. Comm. Stat. Theory Methods. 36, 1855-1866 (2007)

Glänzel, W. A characterization theorem based on truncated moments and its application to some distribution families. In Mathematical statistics and probability theory, Springer, Dordrecht. 75-84(1987)

Gradshteyn, I.S., Ryzhik, I.M.: Table of Integrals, Series, and Products. Academic press (2014)

Jayakumar, K., Mathew, T:: On a generalization to MarshallOlkin scheme and its application to Burr type XII distribution. Stat. Pap. 49, 421-439 (2008)

Jose, K. K., Naik, S. R., \& Ristić, M. M. Marshall-Olkin q-Weibull distribution and max-min processes. Statistical papers, 51(4), 837-851(2010)

Krishna, E., Jose, K. K., Alice, T., \& Ristić, M. M. The Marshall-Olkin Fréchet distribution. Communications in Statistics-Theory and Methods, 42(22), 4091-4107(2013).

Korkmaz, M.Ç., Cordeiro, G.M., Yousof, H.M., Pescim, R.R., Afify, A.Z., Nadarajah, S.: The Weibull Marshall-Olkin family: regression model and application to censored data. Commun Stat-Theory Methods. 48(16), 4171-4194 (2019).

Lee, E.T., Wang, J.W.: Statistical Methods for Survival Data Analysis, 3rd edn, p. MR1968483. Wiley, New York (2003)

Marshall, A.W., Olkin, l.: A new methods for adding a parameter to a family of distributions with application to the exponential and Weibull families. Biometrika. 84, 641-652 (1997)

Nadarajah, S., Jayakumar, K., Ristic, M.M.: A new family of lifetime models. J. Stat. Comput. Simul. 83, 1389-1404 (2013)

Ristić, M. M., \& Kundu, D. Marshall-Olkin generalized exponential distribution. Metron, 73(3), 317-333(2015).

Saboor, A., \& Pogány, T. K. Marshall-Olkin gamma-Weibull distribution with applications. Communications in Statistics-Theory and Methods, 45(5), 1550-1563(2016).

Santos-Neto, M., Bourguignon, M., Zea, L.M., Nascimento, A.D., Cordeiro, G.M.: The Marshall-Olkin extended Weibull family of distributions. J. Stat. Distrib. Appl. 1, 1-24 (2014)

Tahir, M.H., Cordeiro, G.M., Alzaatreh, A., Mansoor, M., Zubair, M.: The logistic-X family of distributions and its applications. Commun. Stat-Theory Methods. 45(24), 7326-7349 (2016)

Yousof, H.M., Afify, A.Z., Nadarajah, S., Hamedani, G., Aryal, G.R.: The Marshall-Olkin generalized-G family of distributions with applications. Statistica. 78(3), 273-295 (2018)

\section{Publisher's Note}

Springer Nature remains neutral with regard to jurisdictional claims in published maps and institutional affiliations. 